\title{
Dynamic and complex optical patterns from colloids of cholesteric liquid crystal droplets
}

\author{
JungHyun $\mathrm{Noh}^{a}$, Irena Drevensek-Olenik ${ }^{b}$, Jun Yamamoto ${ }^{c}$ and Jan P. F. Lagerwall ${ }^{a}$ \\ ${ }^{a}$ University of Luxembourg, Physics \& Materials Science Research Unit, L-1511 Luxembourg, \\ Luxembourg; \\ ${ }^{b}$ University of Ljubljana, Faculty of Mathematics and Physics, SI 1000 Ljubljana, Slovenia ${ }^{c}$ \\ Kyoto University, Department of Physics, Kyoto 606-8502, Japan
}

\begin{abstract}
Drops or shells of a planar-aligned short-pitch cholesteric liquid crystal exhibit unique optical properties due to the combination of Bragg reflection in the cholesteric helix and a radial orientation of the helix axis. If such a droplet is illuminated from above, light is reflected into a continuous set of cones, the opening angles of which depend on where on the droplet the light hits its surface. For the wavelength that fulfills the Bragg condition the reflection is dramatically enhanced, yielding the light cones colored. A photonic cross communication scheme arises for certain angles, reflecting light back to the observer from a different droplet than the one originally illuminated. This gives rise to an intricate pattern of colored and circularly polarized spots. A number of interesting applications may be developed based on this pattern, e.g. in identification and authentication devices. We have carried out a detailed spectrophotometric analysis of the patterns, localized to individual spot maxima. A quantitative comparison between the measured spectra and the reflection wavelength expected from a model for the pattern generation allows us to conclude that the droplets are in fact not spherical but slightly ellipsoidal.
\end{abstract}

Keywords: Cholesteric liquid crystals, colloidal crystals, selective reflection, Bragg reflection, photonics, pattern formation, microfluidics

\section{INTRODUCTION}

The striking beauty as well as the extraordinary responsiveness of cholesteric liquid crystals have made them among the most studied and best understood classes of liquid crystal, and few are those liquid crystal textbooks or courses that miss the opportunity to teach the origin of their iridescent colors. The ease with which these liquids self-organize into a long-range ordered helical arrangement, ${ }^{1}$ giving rise to Bragg reflection of circularly polarized light that is often in the visible range, turns cholesterics into a unique class of photonic materials, ${ }^{2}$ exhibiting optical properties that are far from trivial to achieve artificially in a top-down-derived structure. Yet, commercialized products utilizing cholesterics are still rather few, typically low-cost, low-key devices like simple thermometers, the most advanced application probably being the pressure-sensitive monocolor display developed by Kent Display for their sketching pads*. Cholesterics thus clearly constitute a readily available materials class with untapped potential. This is beginning to be explored more vividly during recent years, e.g. in cellulosebased lyotropic cholesterics dried to paper-like iridescent films ${ }^{3-6}$ with potential e.g. for optical encryption, ${ }^{7}$ or in liquid crystal-based tunable lasers. ${ }^{8-18}$

An interesting example is the case of spherical liquid crystal samples, ${ }^{9,14}$ i.e. a cholesteric brought into droplet or shell morphology as a component of an emulsion. When applied in a laser such a geometry offers 3D omnidirectional lasing in addition to the tunability aspect. Because of the radial arrangement of the cholesteric helix the usual dependence of the color on the viewing angle does not appear: in any direction that you view a planar-aligned cholesteric droplet you are looking along the helix axis. On the other hand, this also means that you see the reflection color only in a central spot in an isolated droplet. The rest appears dark, in contrast to a flat sample which is colored over the full surface.

Further author information: (Send correspondence to J.P.F.L.)

J.P.F.L.: E-mail: jan.lagerwall@lcsoftmatter.com, Telephone: +352 4666446219

${ }^{*}$ See http://www.kentdisplays.com .

Emerging Liquid Crystal Technologies X, edited by Liang-Chy Chien, Harry J. Coles, Hirotsugu Kikuchi, Ivan I. Smalyukh, Proc. of SPIE Vol. 9384, 93840T - (c) 2015 SPIE · CCC code: 0277-786X/15/\$18 · doi: 10.1117/12.2180516 

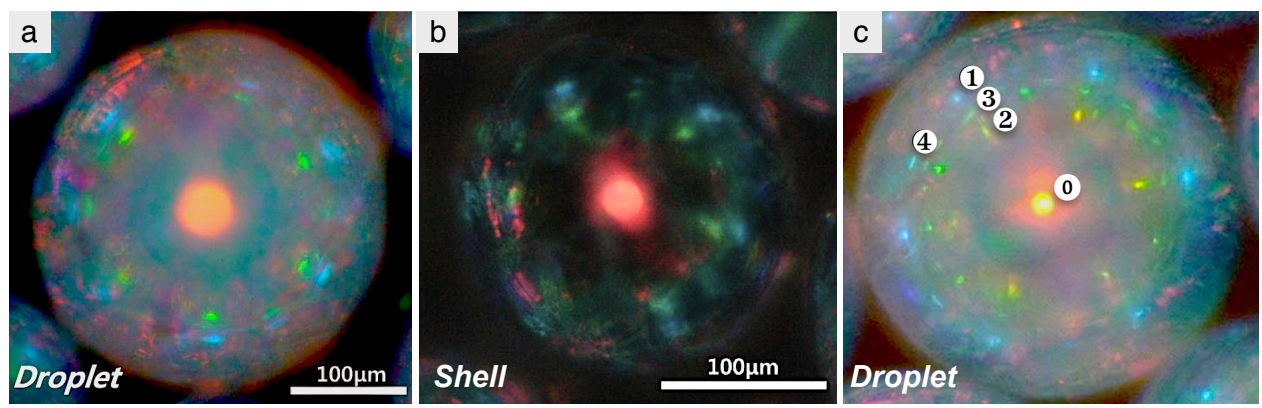

Figure 1. Reflection patterns from planar-aligned droplets (a) and shells (b) of a cholesteric liquid crystal with pitch in the range of generating red selective reflection (central, slightly overexposed, spot), placed in a colloidal crystal arrangement together with a large number of other identical droplets/shells that surround the pictured sphere. Pane (c) shows the central droplet in an arrangement of larger droplets (about $400 \mu \mathrm{m}$ in diameter), displaying a larger number of spots of various types, which are also slightly sharper. The numbers up to 3, displayed next to spots along a radius directed towards the nearest neighbor $(n n)$ droplet, identify the origin of each spot, using the same number coding as in Fig. 2. That figure shows a schematic representation of the cross section along the same radius. Spot number 4 is not represented in Fig. 2 since it originates in direct photonic communication between next nearest neighbor ( nnn) droplets, thus in a different cross section plane than in Fig. 2.

In a recent paper ${ }^{19}$ we reported the observation and explanation of a set of novel and very interesting optical properties that arise in an intermediate situation, when a large number of planar-aligned cholesteric droplets or shells of uniform size are brought into a 2D colloidal crystal arrangement, thus covering a surface that can be arbitrarily large and of arbitrary shape, with a unique photonic response at any location in the surface. This situation arises because the cholesteric droplets/shells in such an arrangement reflect light not just straight back to the observer from the spot at their centers, but also between each other, sometimes over distances considerably further than an individual droplet diameter, before the light is (for some angles) eventually reflected back to the observer, cf. Fig. 1. As seen in panes (a) and (b) of the figure, the reflection pattern arising from this photonic cross communication is qualitatively identical for droplets and shells. For simplicity we will be speaking about droplets in the following, but the reader should keep in mind that the same behavior is observed for shells with planar alignment on the in- as well as outside. The generated pattern is dynamically tunable, the details depending on the illuminated area, the pitch of the cholesteric helix, the sizes and exact arrangement of the droplets, the density of the droplets compared to that of the continuous phase, as well as on any deformation from perfect spherical shape that the droplets are subject to. Fig. 1c shows an example from an arrangement of rather large droplets, displaying a larger number of spots with different colors and at different locations within the central droplet. These patterns open up for new approaches to applications of cholesterics e.g. in security pattern generation or in sensors. ${ }^{19}$

In this paper we present a quantitative spectrophotometric investigation of the spots making up the patterns. By comparing the measured wavelength for each series of spots with a previously presented model, summarized below and described in full detail in our previous paper, ${ }^{19}$ we conclude that the droplets are not spherical but ellipsoidal, as sketched in Fig. 2. We believe that the origin of this deformation is the same buoyancyoriginated force that drives the droplets into a colloidal crystal arrangement, in combination with the fact that the continuous phase is not a bulk sample with flat surface, but a finite droplet deposited on a substrate with non-zero contact angle.

\section{SUMMARY OF THE PREVIOUS MODEL}

In our previous paper ${ }^{19}$ we provided the qualitative explanation for the patterns seen in these colloidal crystals of cholesteric liquid crystal droplets, schematically summarized in Fig. 2. Compared to the corresponding figure in our earlier paper this figure has been modified in the respect that the droplets are now ellipsoids rather than perfect spheres. This drawing is thus more realistic for the samples studied here, although different sample preparation may yield situations where the droplets are very close to perfectly spherical, or even distorted in the 


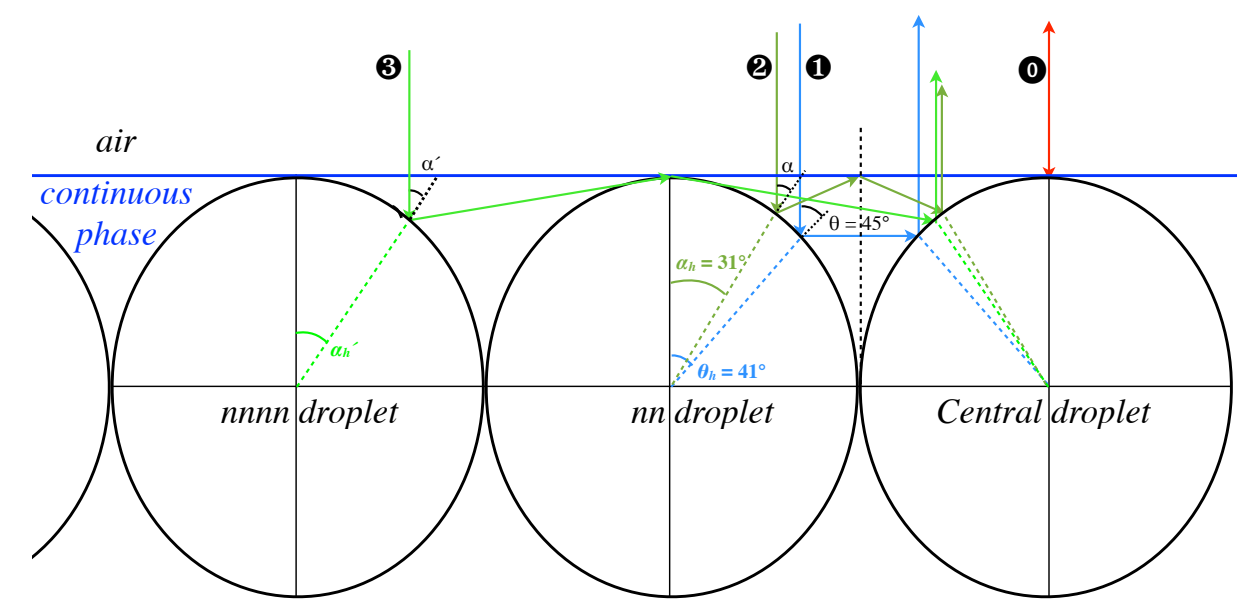

Figure 2. Schematic drawing of the photonic communication paths in the cross section plane along the nearest-neighbor $(n n)$ radius with spots numbered $0-3$ in Fig. 1c. The ordinary selective reflection along the helix axis is indicated with the label 0 , the direct $n n$ communication path is labelled 1 . The total internal reflection (TIR)-mediated communication paths indicated are (2) between nearest neighbors and (3) between next next nearest neighbors (nnnn). The slight deviation from a perfectly flat surface of the continuous phase that may be a result of the buoyancy of the less dense liquid crystal droplets is ignored. Angles without indices refer to droplet surface normals, angles with the index $h$ refer to the local inclination of the cholesteric helix axis inside the droplet. Because the nnnn communication spots were not analyzed with the spectrophotometer we cannot give a value for the angle $\alpha_{h}^{\prime}$.

perpendicular direction. The droplet shape has an impact on the pattern that is generated, because each spot except the ordinary selective reflection at the center of each droplet is generated by a more or less complex light path that involves reflections at the interface between the droplet and the continuous phase.

The ordinary selective reflection is indicated with 0 in Fig. 1c and Fig. 2. As demonstrated and discussed in our previous paper ${ }^{19}$ this is the only spot to be seen in the central droplet if only this droplet is illuminated. If the illumination area is gradually expanded to encompass also surrounding droplets, by opening the field aperture, additional series of spots appear, one after another. ${ }^{19}$ The first additional series is the one indicated with the number 1 in Fig. 1c and Fig. 2. It arises because light from above hits a droplet with an angle of $\theta=45^{\circ}$ with respect to its surface normal, yielding a reflection that is perfectly horizontal, thus hitting the nearest neighbor $(n n)$ droplet at the same height, where it will again be reflected at the same angle with respect to the surface normal, leading to the light going back in the vertical direction, thus reaching the observer's eyes or camera. This reflection is however greatly intensified for one particular wavelength, namely the one that fulfills the conditions for Bragg reflection in the cholesteric helix, giving the spot a distinct color. For a spherical droplet the angle of Bragg reflection would be identical to the angle the light makes with the surface normal, thus $45^{\circ}$. However, as we will show below the wavelength that is reflected does not correspond to $45^{\circ}$ angle Bragg reflection, indicating that the droplets are in fact slightly deformed ellipsoidally, as indicated in Fig. 2. This deviation from a spherical shape will give an analogous wavelength shift also for the other spots, which are all due to photonic communication paths that involve a total internal reflection (TIR) event at the continuous phase-air surface. If the TIR event takes place exactly in between two $n n$ droplets we get the path labelled 2 in Fig. 2, yielding the spot labelled 2 in Fig. 1c. If it instead occurs at the top of the $n n$ droplet, it mediates communication between next next nearest neighbors (nnnn), following path 3 in Fig. 2, giving rise to spot 3 in Fig. 1c.

\section{EXPERIMENTAL}

\subsection{Materials}

The cholesteric $\left(\mathrm{N}^{*}\right)$ liquid crystal mixtures were composed of the commercial nematic mixture RO-TN 615 from Roche (Switzerland) and the chiral dopant (S)-4-Cyano-4'-(2-methylbutyl)biphenyl (CB15), purchased from 

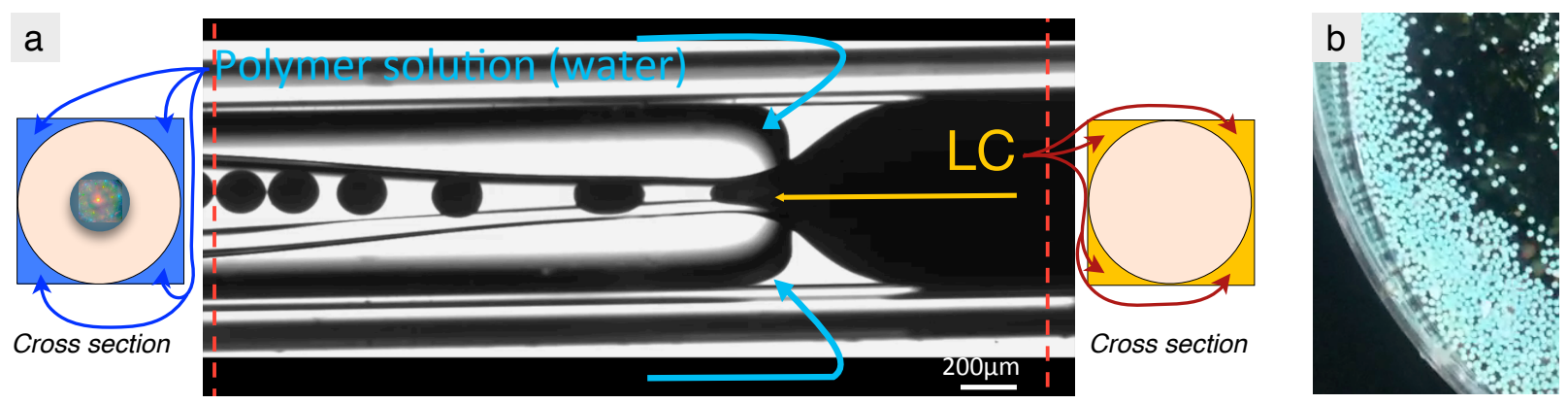

Figure 3. (a) High-speed video image of the microfluidic droplet production, with cross-section drawings on the left and right illustrating where the continuous phase, the liquid crystal (LC) and the droplets are found in the nested capillary setup. The right side of the set-up contains an inner tapered cylindrical capillary which is not used to flow any material (the circle on the right cross-section drawing is empty) but it is needed for ensuring a correct flow focusing of the liquid crystal stream from the right into the cylindrical internal collection tube on the left (circle in the left cross-section drawing). (b) A petri dish with rather large droplets (about $1 \mathrm{~mm}$ in diameter) of a short-pitch cholesteric liquid crystal mixture (40\% CB15) with blue-green selective reflection at normal incidence.

Synthon Chemicals (Germany). The mixtures can be tuned to reflect from the infrared to the ultraviolet range, with visible normal-incidence reflection occurring for CB15 concentrations between 30 wt.- $\%$ and 50 wt.-\% (the pitch gets shorter the higher the chiral dopant concentration). For ensuring planar alignment and counteracting coalescence of the liquid crystal droplets, they were dispersed in a continuous phase consisting of a distilled water + glycerol mixture (50/50 volume ratio) in which 3 wt.-\% polyvinylalcohol (PVA) was dissolved (Sigma-Aldrich, $\mathrm{M}_{w} \approx 31,000-50,000 \mathrm{~g} \mathrm{~mol}{ }^{-1}, 98-99 \%$ hydrolysed).

\subsection{Microfluidic droplet production}

The droplets discussed in this paper were produced using the capillary microfluidics approach for preparing multiple emulsions originally reported by Utada et al. ${ }^{20}$ and first applied to liquid crystals by Fernandez-Nieves et al. ${ }^{21}$ The same set-up can be used to make shells, by flowing an internal phase within the liquid crystal stream, but in the following only the results obtained with droplets (no internal phase) are discussed. A high-speed video image of the process, with overlaid explanatory drawings, is shown in Fig. 3a. The liquid crystal is flown from the right in the four corners of a capillary with square cross section, surrounding a cylindrical capillary with tapered end (this carries the internal phase when shells are desired). From the left in the same square capillary is flown the aqueous continuous phase, immiscible with the liquid crystal, also in the four corners surrounding a cylindrical capillary, this time with a blunt end. The liquid crystal is flow focused close to the opening of the blunt capillary, by the counter-flowing aqueous continuous phase, into the blunt capillary which acts as a collection channel for the cholesteric droplet emulsion. The macroscopic emulsion in case of very thick droplets (about $1 \mathrm{~mm}$ in diameter) of a cholesteric with pitch short enough to give blue-green selective reflection along the helix is shown in Fig. 3b. The continuous water-glycerol-PVA phase ensures planar alignment of the director at the droplet surface, thus a radial orientation of the cholesteric helix.

\subsection{Bringing droplets into a colloidal crystal arrangement}

After production of the emulsion with the cholesteric droplets this was collected in a petri dish, as shown in Fig. 3b. For investigation in a microscope and using the spectrophotometer a small volume from such an emulsion (with smaller droplets of a different cholesteric mixture than what is shown in Fig. 3b) was deposited on a silicon chip on which the emulsion made a non-zero contact angle. Since the cholesteric liquid crystal has a lower density than the continuous phase, the droplets float up towards the top of the resulting (close to) hemispherical droplet, as illustrated in Fig. 4. Because of the non-zero curvature of the surface of such a small volume of continuous phase, the normal force that opposes the buoyancy force once the droplets reach the surface then has a horizontal component. This has the benefit that it drives the droplets into the close-packed hexagonal colloidal crystal arrangement. However, this force can also lead to a deformation of the droplets if they are soft 


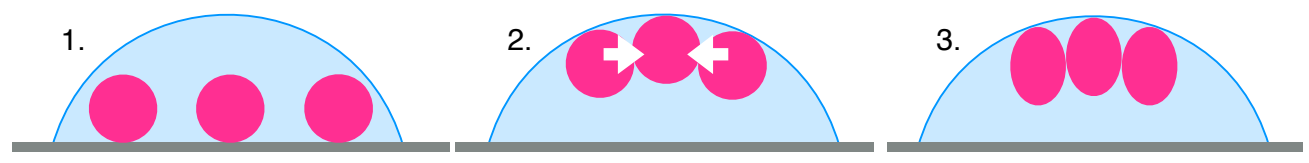

Figure 4. A small droplet of the emulsion containing the cholesteric droplets (pink) was deposited on a silicon substrate on which the aqueous continuous phase (blue) makes a non-zero contact angle. The continuous phase thus makes a close to hemispherical volume, through which the cholesteric droplets, due to their lower density compared to the continuous phase, float up towards the top. Because of the curvature of the continuous phase surface the buoyancy produces an effective force that also has a horizontal component (white arrows) that pushes the droplets together. This would explain the slight ellipsoidal deformation.

enough. As described below the spectrophotometric data suggest that a deformation into an ellipsoidal shape indeed took place.

\subsection{Optical characterization}

The samples on silicon chips were placed in an Olympus polarizing microscope operating in reflection mode, allowing observation by eye or a monochrome camera. The microscope was equipped with an Andor Technology PLC spectrophotometer, allowing the spectrum within a $200 \mathrm{~nm}$ wide window to be obtained for each of 496 pixels along one line in the image, defined by a slit of variable width. Because the spectral width of interest is the full visible range, multiple spectra were obtained for each slit orientation selected within a sample, shifting the center wavelength between measurements such that the full range of interest was covered by the combined experiments. For each slit orientation the spectra were then merged in a data plotting software (Pro Fit). Due to the automatic intensity regulation of the spectrophotometer, changing the absolute intensity values from one partial spectrum to the next, there was generally a mismatch in intensity at the splicing points which we tried to minimize by normalization of the data to a common base level. In addition, thermal motion led to subtle displacements of droplets between measurements, leading to slight mismatch also in pixels. As the absolute intensities are of no importance, only the wavelengths of peaks, and as all spots cover a range of at least about 10 pixels of the spectrophotometer, these issues do not cause a real problem for the analysis, but they do lead to some artifacts in the spectra at splicing points.

For each sample two slit orientations were studied, one for analyzing the nearest neighbor $(n n)$ communication spots, another for the next nearest neighbor $(n n n)$ spots. The slit width and orientations are shown for the sample discussed in detail in this paper in Fig. 5. The field aperture opening was such that no next next nearest neighbor (nnnn) spots were visible.

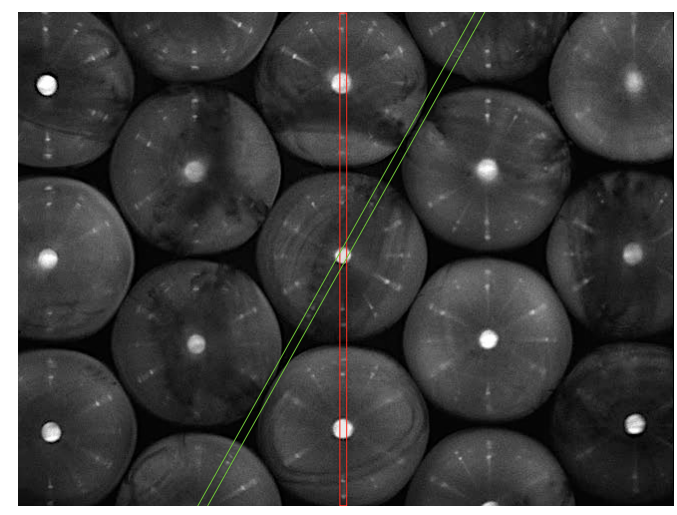

Figure 5. Monochrome polarizing microscopy photo of the sample of droplets with 33 wt.- $\%$ chiral dopant in the cholesteric mixture. The red box indicates the slit used during spectrophotometric analysis of the nearest neighbor $(n n)$ communication spots, the green the slit for analyzing the next nearest neighbor ( $n n n)$ spots. 


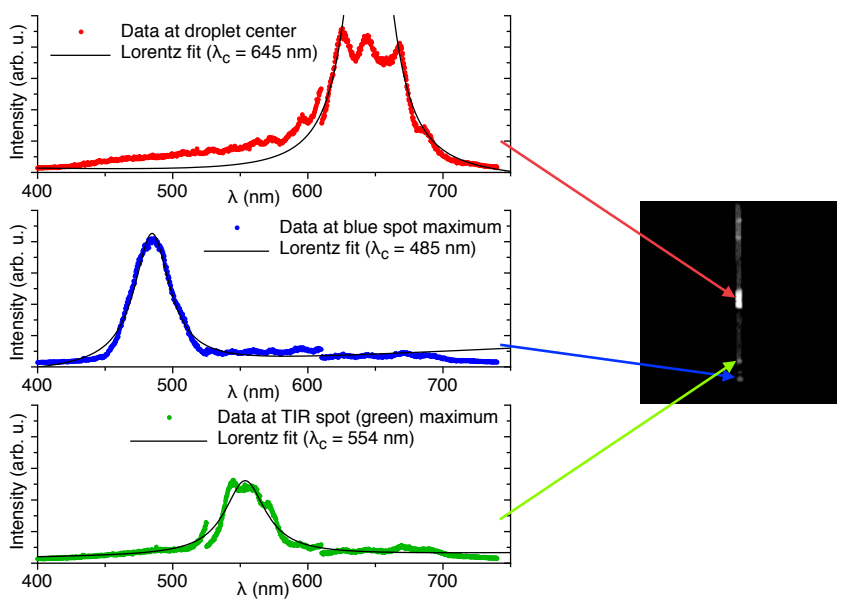

Figure 6. Spectra obtained along the nearest neighbor $(n n)$ communication direction in the central droplet. The top spectrum was obtained at the center of the ordinary selective reflection spot, the middle spectrum in the spot arising from direct $n n$ communication, and the bottom spectrum was measured in the spot originating from TIR-mediated $n n$ communication. The photo on the right shows the sample texture through the slit used during the measurements, with arrows linking each spectrum to the spot in which it was measured.

\section{RESULTS AND DISCUSSION}

For quantitative analysis of the wavelengths reflected in the different spots of the pattern we investigated spectra obtained with droplets of the cholesteric mixture with 33 wt.- $\%$ chiral dopant. We chose this mixture because all spots of the pattern are in the visible range, and are thus suitable for investigation with the spectrophotometer used. The mixture displays red selective reflection at normal incidence, hence the central spot in the droplets appears red. The peripheral spots derived from direct inter-droplet communication appear blue, whereas those spots that arise from TIR-mediated communication have colors in the green-yellow range.

We first discuss the analysis of the spots arising from direct and TIR-mediated photonic communication with the nearest neighbor $(n n)$ droplets, in comparison with the central ordinary selective reflection spot. To this end we studied the spectra obtained in each pixel along the slit indicated by the red box in Fig. 5. Three representative spectra are shown in Fig. 6, corresponding to the maximum intensity pixel within each spot of interest (corresponding to spots 0, 1 and 2 in Fig. 1c and Fig. 2) in the center droplet. A single-peak Lorentz function was fitted to the main peak of each spectrum in order to obtain the central wavelength. The Lorentz function fits relatively well to the peripheral spots, while it is not truly appropriate for the regular selective reflection spot (central spot). Here the full width of the selective reflection band is visible in the spectrum, with an overlaid modulation which may be due to interference with light reflected at the surface of the continuous phase, but by fitting only to the left and right flanks of the peak a reliable value of the central wavelength $\lambda_{c}$ could be obtained.

The wavelength of the central spot is measured to be $645 \mathrm{~nm}$, corresponding well to its red color. For the direct inter-droplet communication spots, whether these originate from communication with the nearest or next nearest neighbors, we would then expect a wavelength of $645 \cos 45^{\circ}=456 \mathrm{~nm}$ in case the droplets are spherical. This is because the direct photonic communication takes place via reflections at the droplet surfaces under the angle $45^{\circ}$, first redirecting the vertically incoming light beam to the horizontal direction, then after a second reflection in the neighbor droplet redirecting it back up vertically. ${ }^{19}$ However, as seen in the second spectrum in Fig 6 the wavelength is considerably longer, $485 \mathrm{~nm}$. This wavelength would arise from Bragg reflection at a slightly smaller angle, $\arccos 485 / 645 \approx 41^{\circ}$. The same discrepancy between measured wavelength and the wavelength calculated based on the spherical droplet model is found for the TIR-mediated communication spot, expected to be at $645 \cos 34^{\circ}=535 \mathrm{~nm}$ (the derivation of the reflection angle $\alpha=34^{\circ}$ can be found in our previous paper ${ }^{19}$ ) but in practice having a peak wavelength of $554 \mathrm{~nm}$. Again, a slightly smaller angle of Bragg 

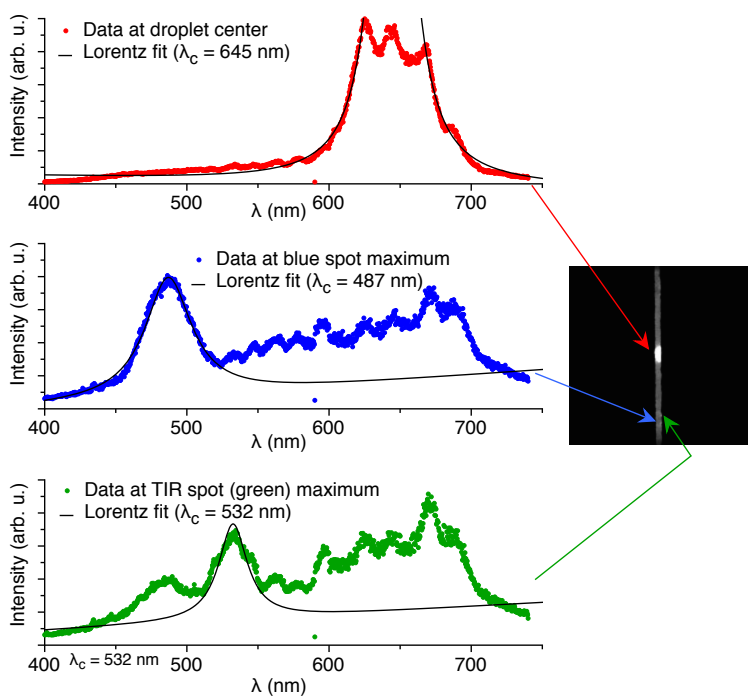

Figure 7. Spectra obtained along the next nearest neighbor (nnn) direction. The top spectrum is obtained in the central regular selective reflection spot, the middle spectrum corresponds to direct nnn communication and the bottom spectrum is for the TIR-mediated nnn communication. The photo on the right shows the sample texture through the slit used during the measurements, with arrows linking each spectrum to the spot in which it was measured.

reflection would explain this wavelength, $\arccos 554 / 645 \approx 31^{\circ}$. The discrepancy is quantitatively identical for both peripheral spots, as $41 / 45 \approx 31 / 34 \approx 0.91$.

To explain this discrepancy we consider which approximations have been made in the model described above, and which may not be valid. First, one may consider that the surface of the continuous phase to the surrounding air is not perfectly flat, as it has been drawn in Fig. 2. Indeed, some deviation is expected due to the buoyancy of the cholesteric droplets, less dense than the continuous phase. However, the slightly different light paths that would result from a continuous phase surface that is curved in response to the droplets pushing upwards would give rise to larger Bragg angles and this can thus not be the explanation to the discrepancy.

Instead, we believe that the discrepancy has its origin in a deviation of the droplet shape from perfectly spherical into an ellipsoidal shape, with a vertical axis that is longer than the horizontal one, as drawn in Fig. 2. As discussed above and visualized in Fig. 4 such a deviation can be expected from the combination of an upwards directed buoyancy force in combination with a curved continuous phase surface, yielding a normal force with a horizontal component that not only pushes the droplets into a close-packed hexagonal arrangement, but even squeezes the liquid droplets slightly, extending them along the vertical axis and compressing them in the horizontal plane. As illustrated in Fig. 4 this droplet deformation would explain the optical data, because even when the reflection angle at the outside droplet surface is $\theta=45^{\circ}$ and $\alpha=34^{\circ}$, respectively, the cholesteric helix axis is slightly less inclined, setting the Bragg angle to the somewhat smaller values $\theta_{h}$ and $\alpha_{h}$ that give rise to the wavelength increase, compared to the values expected for spherical droplets.

We also analyzed the spots along the nnn communication direction, indicated with a green box in Fig. 6 . These spots were weaker and the corresponding spectra considerably noisier, but the peaks were still distinct enough to allow determination of the peak wavelength by fitting a Lorentz peak to the data, cf. Fig 7 . For the ordinary selective reflection peak we obtained the exact same central wavelength and for the direct $n n n$ communication the wavelength was identical to that of direct $n n$ communication within $2 \mathrm{~nm}$, thus within experimental error. The wavelength of the TIR-mediated nnn communication yields a Bragg reflection angle of $34^{\circ}$. For spherical droplets this angle would have been expected for TIR-mediated $n n$ communication while for TIR-mediated communication over longer distances the angle should continuously increase up to a limiting value of $45^{\circ}$ (which yields horizontal reflection and thus no TIR event at any distance). ${ }^{19}$ The wavelength of the TIR-mediated nnn communication spot thus corroborates qualitatively the conclusion that the droplets are ellipsoidally deformed. 


\section{CONCLUSIONS}

Based on a localized spectrophotometric analysis of the various spots of the patterns arising from photonic cross communication between cholesteric liquid crystal droplets brought into a close-packed hexagonal colloidal crystal arrangement, we could corroborate our previous model for the origin of the patterns, with the modification that the droplets in the studied samples are not spherical, but ellipsoidal. We propose that this deformation arises from the buoyancy force driving the liquid crystal droplets, slightly less dense than the continuous phase, up towards a continuous phase surface that curves. A horizontal normal force component then brings the droplets into the desired close-packed arrangement, but it also induces a slight deformation from spherical shape. The dynamic quality of the patterns and the sensitive dependence on droplet arrangement, shape and cholesteric pitch gives these patterns a unique quality that may find various uses, e.g. in security patterns. ${ }^{19}$

\section{ACKNOWLEDGMENTS}

Financial support from the National Research Foundation (NRF, Korea), grant number 490-20110016, and NRF-JSPS (Japan) bilateral project, grant number 490-20130024, is gratefully acknowledged.

\section{REFERENCES}

[1] Lagerwall, J. P. F. and Scalia, G., "A new era for liquid crystal research: Applications of liquid crystals in soft matter nano-, bio- and microtechnology," Curr. Appl. Phys. 12(6), 1387-1412 (2012).

[2] Kitzerow, H., "Tunable photonic crystals," Liq. Cryst. Today 11(4), 3-7 (2002).

[3] Park, J. H., Noh, J., Schütz, C., Salazar-Alvarez, G., Scalia, G., Bergström, L., and Lagerwall, J. P. F., "Macroscopic control of helix orientation in films dried from cholesteric liquid-crystalline cellulose nanocrystal suspensions," ChemPhysChem 15(7), 1477-1484 (2014).

[4] Lagerwall, J. P. F., Schütz, C., Salajkova, M., Noh, J., Park, J. H., Scalia, G., and Bergström, L., "Cellulose nanocrystal-based materials: from liquid crystal self-assembly and glass formation to multifunctional thin films," NPG Asia Mater 6(1), e80 (2014).

[5] Habibi, Y., "Key advances in the chemical modification of nanocelluloses.," Chem. Soc. Rev. 43(5), 1519$1542(2014)$.

[6] Klemm, D., Kramer, F., Moritz, S., Lindstrom, T., Ankerfors, M., Gray, D., and Dorris, A., "Nanocelluloses: A new family of nature-based materials," Angew Chem Int Edit 50(24), 5438-5466 (2011).

[7] [Nanocrystalline cellulose for covert optical encryption], 8258, SPIE-INT SOC OPTICAL ENGINEERING, Zhang, YP (reprint author), McGill Univ, Dept Elect \& Comp Engn, Montreal, PQ, Canada [Zhang, Yu Ping; Chodavarapu, Vamsy P.; Kirk, Andrew G.] McGill Univ, Dept Elect \& Comp Engn, Montreal, PQ, Canada. (2012).

[8] Wenzlik, D., Varanytsia, A., Munoz, A., Kosa, T., Taheri, B., Zentel, R., and Palffy-Muhoray, P., "Distributed feedback lasing in cellulose films," Opt. Mater. Express 4(1), 162 (2014).

[9] Uchida, Y., Takanishi, Y., and Yamamoto, J., "Controlled fabrication and photonic structure of cholesteric liquid crystalline shells," Adv. Mater. 25(23), 3234-3237 (2013).

[10] Munoz, A., McConney, M. E., Kosa, T., Luchette, P., Sukhomlinova, L., White, T. J., Bunning, T. J., and Taheri, B., "Continuous wave mirrorless lasing in cholesteric liquid crystals with pitch gradient across the cell gap," Opt. Lett. 37(14), 2904-2906 (2012).

[11] Choi, H., Nishimura, S., Toyooka, T., Ishikawa, K., and Takezoe, H., "Analysis of cavity-mode lasing characteristics from a resonator with broadband cholesteric liquid-crystal bragg reflectors," Adv. Funct. Mater. 21(18), 3430-3438 (2011).

[12] Takanishi, Y., Ohtsuka, Y., Suzaki, G., Nishimura, S., and Takezoe, H., "Low threshold lasing from dyedoped cholesteric liquid crystal multi-layered structures," Opt. Express 18(12), 12909-12914 (2010).

[13] Coles, H. and Morris, S., "Liquid-crystal lasers," Nat. Photonics 4(10), 676-685 (2010).

[14] Humar, M. and Musevic, I., "3d microlasers from self-assembled cholesteric liquid-crystal microdroplets," Opt. Express 18(26), 26995-27003 (2010). 
[15] Chanishvili, A., Chilaya, G., Petriashvili, G., Barberi, R., Bartolino, R., Cipparrone, G., Mazzulla, A., and Oriol, L., "Phototunable lasing in dye-doped cholesteric liquid crystals," Appl. Phys. Lett. 83(26), 5353-5355 (2003).

[16] Shibaev, P., Kopp, V., Genack, a., and Hanelt, E., "Lasing from chiral photonic band gap materials based on cholesteric glasses," Liq. Cryst. 30(12), 1391-1400 (2003).

[17] Funamoto, K., Ozaki, M., and Yoshino, K., "Discontinuous shift of lasing wavelength with temperature in cholesteric liquid crystal," Jpn. J. Appl. Phys. 42(12B), L1523-L1525 (2003).

[18] Finkelmann, H., Kim, S., Munoz, A., Palffy-Muhoray, P., and Taheri, B., "Tunable mirrorless lasing in cholesteric liquid crystalline elastomers," Adv. Mater. 13(14), 1069-1072 (2001).

[19] Noh, J., Liang, H.-L., Drevensek-Olenik, I., and Lagerwall, J. P. F., "Tuneable multicoloured patterns from photonic cross communication between cholesteric liquid crystal droplets," J. Mater. Chem. C 2(5), 806-810 (2014).

[20] Utada, A., Lorenceau, E., Link, D. R., Kaplan, P. D., Stone, H. A., and Weitz, D. A., "Monodisperse double emulsions generated from a microcapillary device," Science 308(5721), 537-541 (2005).

[21] Fernandez-Nieves, A., Vitelli, V., Utada, A., Link, D. R., Marquez, M., Nelson, D. R., and Weitz, D. A., "Novel defect structures in nematic liquid crystal shells," Phys. Rev. Lett. 99(15), 157801 (2007). 